\title{
Theme: Pediatric Pulmonology
}

Q

Tiotropium add-on therapy in adolescents with moderate asthma (J Allergy Clin Immunol. 2016;138:441-50)

In a 48-week, Phase III double-blind, placebo-controlled, parallelgroup study conducted on 398 adolescents aged 12-17 years from 65 sites in 12 countries, patients were randomized 1:1:1 into $5 \mu \mathrm{g}, 2.5 \mu \mathrm{g}$ or placebo groups. The authors reported an improvement in their primary endpoint of peak Forced Expired Volume in 1 second (FEV1) at 24 weeks, and the lowest FEV1 (trough FEV1) was significantly improved in the $5 \mu \mathrm{g}$ dose group. Asthma control and health-related quality of life showed a trend towards improvement over the 48-week period. The incidence of adverse events was comparable across the three groups. This trial provides evidence that supports the use of long-acting anticholinergic agents such as tiotropium in patients with moderate persistent asthma that remained poorly controlled despite therapy with long-acting beta agonists (LABAs), if there is development of tolerance, or if there are safety concerns with the use of LABAs. As more studies provide additional evidence for Tiotropium use, it has a potential to be incorporated into the asthma management plans for adolescents with moderate persistent asthma with poor control.

The airway microbiome at birth (Sci Rep. 2016;6:31023)

The human lung microbiome is an important area of research, as specific alterations in airway microbiota have been associated with many disease states such as asthma, cystic fibrosis and chronic obstructive pulmonary disease. This study examined the airway microbiome of preterm and term infants, and compared it to those with bronchopulmonary dysplasia (BPD) using sequencing of the $16 \mathrm{~S}$ ribosomal RNA genes. The authors reported a diverse yet similar airway microbiome at birth in both preterm and term infants, but these were different from the less diverse microbiome seen in older preterm infants with established BPD. They identified a temporal dysbiosis of the airway microbiome in extremely low birth weight (ELBW) infants who go on to develop BPD. Interestingly, the airway microbiome of ELBW infants born by vaginal versus caesarian section did not differ significantly, although the genus Lactobacillus was decreased in airway microbiota of those with chorioamnionitis and for those who later developed BPD. Endotoxin concentrations were also noted to be increased in the airways of infants with BPD. Early life gut microbial alterations (the 'gut-lung axis') and the potential interactions between genes of the microbiota and the host may provide further clues to this complex phenomenon that is established very early in life.

Lung function trajectory from childhood to the fourth decade of life (Am JRespir Crit Care Med. 2016;194:607-12)

As low lung function in childhood and early adulthood is associated with increased risk of development of chronic obstructive pulmonary disease later in life, it is important to understand the natural history of progression of lung function changes over time. The cohort of individuals enrolled in the Tucson Children's Respiratory Study which was started in the early 1980s to understand the effect of lung function on wheezing and risk of asthma later in life, have provided important longitudinal data on lung function. Among this nonselected birth cohort of 1246 participants, 599 had 2 or more spirometry results between 11 and 32 years of age, for a total of 2142 observations. The authors were able to identify lung function trajectories that included a) normal lung function throughout, b) persistently low lung function, and c) decline from normal to low lung function later in life. For individuals in the persistently low lung function trajectory, the authors found that they had history of maternal asthma, higher incidence of early life infection with respiratory syncytial virus, and physician diagnosed active asthma at 32 years of age. All of these individuals had evidence of small airway dysfunction in infancy and at 6 years of age as well. The authors concluded that this distinct group of individuals with a low lung function trajectory may have been established at birth and predisposes them to chronic obstructive pulmonary disease later in life.

Obesity and airway dysanapsis in children with and f without asthma (Am J Respir Crit Care Med. 2016;Aug 23 [Epub ahead of print])

Obesity is a risk factor for asthma, and with rising incidence of obesity in urban pediatric populations, it is important to understand its effect on the lung function of children. Airway dysanapsis is defined as the physiologic divergence between the growth of the lung parenchyma and the size of the airways, and can be recognized by spirometry that demonstrates normal Forced Expired Volume in one second (FEV1) and Forced Vital Capacity (FVC), and an abnormal FEV1/FVC ratio. Even though increase in airway length tracks with lung volumes, but airway caliber growth does not. Therefore, dysanapsis can be seen in healthy individuals with expiratory flow limitation and obesity in children has also been associated with reduced FEV1/FVC ratio. The authors evaluated for dysanapsis in obese children by analyzing longitudinal and cross-sectional pulmonary function data from various sources and included populations with and without asthma. The authors defined dysanapsis as normal to high $\mathrm{z}$-score for FVC $\left(>0.674\right.$ or $75^{\text {th }}$ percentile), normal $z$-score for FEV1 $\left(>-1.645,5^{\text {th }}\right.$ percentile or lower limit of normal) and low FEV 1/FVC $(<0.80)$. They found that obesity was associated with dysanapsis both in cross-sectional and longitudinal data sets, and they found higher lung volumes and lower flows, along with ventilation inhomogeneity in these children. For obese children with asthma, they found that dysanapsis was associated with severe disease exacerbations and use of systemic steroids. The authors concluded that obese children show relative airflow obstruction throughout airways of all sizes, and their lung volumes show larger vital capacity, residual volume (RV) and Total Lung Capacity (TLC), but normal RV/TLC ratio suggesting that there is no air trapping. This suggests that dysanapsis or asymmetric growth of lungs and airways can have significant impact on obese children with asthma and may partly explain the decreased response to asthma medications in this group.

LOKESH GUGLANI lokesh.guglani@emory.edu 